\title{
Preface
}

\section{Hepato-centric Musings of Two Intensivists-Back to the Future}
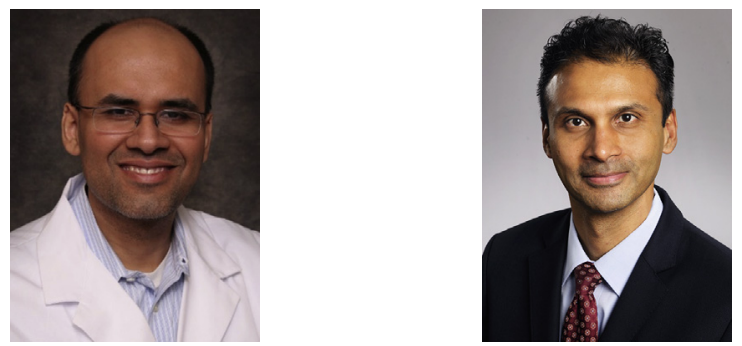

Rahul S. Nanchal, MD, MS, FCCM Ram M. Subramanian, MD, FCCM Editors

The liver as a structure has intrigued humans for perhaps more than 30 millennia. In the ancient world, it was regarded as "the seat of the soul." Galen viewed the body as hepato-centric and considered the liver the origin of veins and the source of blood. The punishment of Prometheus in Greek mythology (artistically depicted as Prometheus Bound [Philadelphia Museum of Art]) for stealing fire from the Gods to give to mankind was to have an eagle tear out and devour his liver each night, which grew back every morning for the process to be repeated. This connotes a remarkable familiarity with the extraordinary regenerative capabilities of the liver. From Hippocrates, who recognized the neuropsychiatric manifestations of advanced liver disease, Ludwig van Beethoven, who underwent repeated paracentesis and eventually succumbed to hepatic coma, Kiernan's description of the hexagonal hepatic lobule, illustrations of Mallory bodies by Frank Burr Mallory in the hepatocytes of patients with alcoholic liver disease, William Bennett Bean's extensive narratives of vascular spiders, to Roberto Groszmann's remarkable insights into the hyperdynamic circulation of liver disease, the literature is rife with a woven tapestry of gradually accumulating evidence that has led to the modern view of liver disease and its complex and fascinating cross-talk with every organ in the body. We stand on the shoulders of giants, and not everyone is acknowledged in this short preface; they are too numerous to tally. Their collective wisdom and seminal observations of the past have paved the way for the present, and more importantly, placed us on the cusp of an incredibly bright future of discovery and therapeutics.

Everyone has a favorite organ; ours is the liver. Two intensivists believe that the liver, often forgotten and frequently neglected, is a master orchestrator of outcomes from critical illness. This is particularly true if the liver is ailing, perhaps more so than any other organ of the body. In this issue, we endeavor to provide the reader with a broad landscape of the far-reaching consequences of hepatic failure on distant organ function, the intricacies of the cross-talk between the liver and multiple organs, and the 
evolution of paradigms of care for the critically ill patient with liver disease. As we reminisce about our chosen path from when seasoned clinicians taught us to recognize the subtle and not so subtle signs of liver disease to the current era of advances in liver support and transplantation, we fondly and joyfully acknowledge the eminent role of our children, Avi, Sanath, and Saurav, in our passions and successes. We are truly back to the future!

Rahul S. Nanchal, MD, MS, FCCM Division of Pulmonary and Critical Care Medicine Froedtert and Medical College of Wisconsin 9200 West Wisconsin Avenue, Suite E5200 Milwaukee, WI 53226, USA

Ram M. Subramanian, MD, FCCM

Emory University 1365 Clifton Road

Building B 6100 Atlanta, GA 30322, USA

E-mail addresses:

rnanchal@mcw.edu (R.S. Nanchal) rmsubra@emory.edu (R.M. Subramanian) 